Żuraw Daria, Oleksa Paulina, Sobczyk Mateusz. Obesity as an "infectious" disease. Journal of Education, Health and Sport. 2021;11(9):534-537. eISSN 2391-8306. DOI http://dx.doi.org/10.12775/JEHS.2021.11.09.069

https://apcz.umk.pl/JEHS/article/view/JEHS.2021.11.09.069

https://zenodo.org/record/5527843

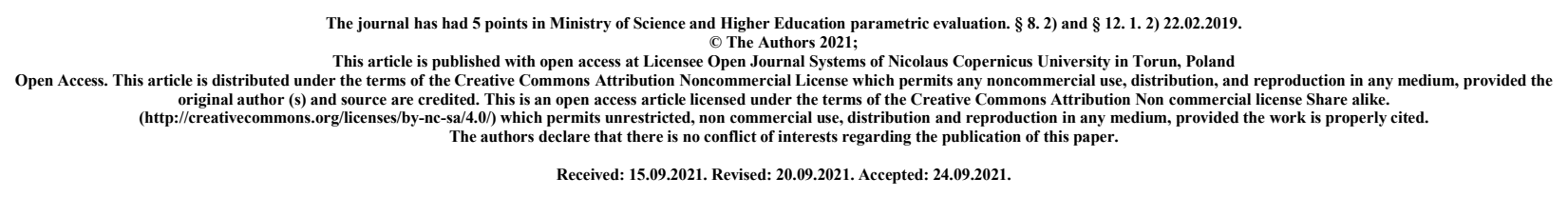

\title{
Obesity as an "infectious" disease
}

\author{
Daria Żuraw ${ }^{1}$, Paulina Oleksa ${ }^{1}$, Mateusz Sobczyk ${ }^{1}$
}

\section{Daria Żuraw, ORCID:0000-0003-0815-4322, dariazuraw19@gmail.com}

Paulina Oleksa, ORCID:0000-0002-3474-6156, gp.oleksa@gmail.com

Mateusz Sobczyk, ORCID:0000-0001-8690-2387, m.sobczyk1777@gmail.com

${ }^{1}$ Student Research Circle at the Department of Epidemiology and Clinical Research Methodology, Medical University of Lublin, Poland

\begin{abstract}
Introduction: Obesity has been recognized as a global epidemic by the WHO, followed by a wealth of empirical evidence supporting its contagiousness. However, the dynamics of the spread of obesity between individuals are rarely studied. A distinguishing feature of the obesity epidemic is that it is driven by a process of social contagion that cannot be perfectly described by the infectious disease model. There is also social discrimination in the obesity epidemic. Social discrimination against obese people plays quite different roles in two cases: on the one hand, when obesity cannot be eliminated, social discrimination can reduce the number of obese people; on the other hand, when obesity is eradicable, social discrimination can cause it to explode.(1)

Material and methods: A literature analysis on obesity epidemic was carried out within the Pubmed, Google scholar and Research Gate platform. The following keywords were used in serach: obesity, epidemy, children, body max index.

Purpose of the work: The aim of the following analysis is to present an obesity as an infectious disease. The steadily increasing percentage of obese people, including children, shows that there is an obesity epidemic. This is the phenomenon of social contagion, which partially explains the concept of homophily, which involves the grouping of people with similar characteristics. Potential explanations are also provided by sharing a living environment with similar access to
\end{abstract}


certain foods and similar opportunities for physical activity, which defines the occurrence of analogous health habits

Key words: obesity; epidemy; children; body max index

\section{Introduction}

Obesity is often defined simply as a condition of abnormal or excessive fat accumulation in adipose tissue, to extent that health may be impaired. The underlying disease is the undesirable positive energy balance and weight gain. However, obese individuals differ not only the amount of excess fat that they store, but also in a regional distribution of that fat within the body. The distribution of the fat included by weight gain affects the risk associated with obesity, and the kinds of disease that results. The graded classification of overweight and obesity: permits meaningful comparisons of weight status within and between populations; makes it possible to identify individuals and groups at increased risk of morbidity and mortality; enables priorities to be identified for intervention at individual and community levels; and provides a firm basis for the evaluation of interventions. Obese individuals with excess fat in the intra-abdominal depots are at particular risk of the adverse health consequences of obesity. Therefore, measurement of waist circumference provides a simple and practical method of identifying overweight patients at increased risk of obesity-aassociated illness due to abdominal fat distribution. Additional tools available for the more detailed characterization of the obese state include methods of measuring body composition, determining the anatomical distribution of body fat, and measuring energy in take and energy expenditure. However, the cost of such techniques and the practical difficulties involved in applying them limit their usefulness to research. (2)

\section{Global meaning of obesity}

Obesity, also known as "globalism," has become a worldwide health problem. Its exponential growth and sometimes deadly risks are generating increasing public awareness, but combating it is no easy task. Understanding its interpersonal dynamics is extremely important. The social environment, and especially social relationships, are closely correlated with human health. The social environment, and especially social relationships, are closely correlated with human health. Lack of social connections can increase the likelihood of death. Many phenomena that spread interpersonally have been found in the social network, such as biological games, indirect reciprocity, misinformation or gossip, and infectious diseases. Obesity is one of them and is often seen as a contagious epidemic. A variety of psychological factors such as depression, selfesteem, insecurity, etc. predispose to obesity but are often overlooked. Because emotions can be transferred directly from one person to another through imitation or "emotional contagion ". In other words, the interpersonal dynamics of the spread of obesity are driven by the process of social contagion. To the extent that obesity is a product of voluntary behavior, the obesity epidemic often results from the spread of undesirable health behaviors, such as overeating, while 
preventing the contagion of healthy behaviors. However, the social contagion of obesity can be reduced or even eliminated by the competitive spread of beliefs about physical activity and beliefs about physical inactivity. Obesity elimination thresholds depend on many factors, such as network topology and clustering of initially obese seeds. Also important is the effect of social discrimination on the obesity epidemic and how it generates different outcomes under different conditions.(3-6)

\section{Description of the examination}

In March 2018, a survey was conducted that included members of the U.S. military professionally assigned to 38 different areas of the United States with their children ages 12-13. Based on observations and surveys, it was noted that being in an environment with more prevalent obesity, compared to areas with less prevalent obesity, was associated with higher average BMI scores and an increased risk of obesity and overweight in the subjects.

Results of the analyses were based on county obesity rate (COR) among adults with linkage to the M-TEEN survey for the area, reflecting the sum of potential factors leading to obesity in each county.

BMI was compared among adults and BMI Z-Score was compared among children. Children's height and weight data were obtained from declarations, but an additional approximately $40 \%$ were measured to verify reported values. In order to highlight the impact of exposure to the area, subjects were divided in terms of time of residence into those living there for less than 24 months and those living there for 24 months or more.

Families assigned to counties with a higher prevalence of obesity were more likely to have increased body weight than families embedded in areas with lower CORs. Similarly, the longer the subjects were incarcerated in areas affected by excess weight gain, the greater the risk of gaining weight. On average, the COR rate was 30\%, with the lowest being in El Paso County, Colorado, at $21 \%$, and the highest at $38 \%$ in Vernon County, Louisiana. With a COR 1\% higher in children, a $4 \%$ increased risk of overweight or obesity was observed. In contrast, in adults, the indicated difference in COR resulted in a 0.08 point increase in BMI and a 5\% higher risk of obesity. It was also confirmed that the association between BMI or BMI Z-score and COR was stronger when families stayed in the area for more than 24 months.

It was most interesting to examine the relationship of the index with eating habits and physical activity, guided by their expected effect on body weight. Respondents were asked to determine how many minutes per week they spent on moderate to vigorous physical activity, and the information obtained was supplemented with dietary interviews about their daily habits. They found that higher COR was associated with less time spent on intense exercise per week in both age groups, as well as more frequent eating of prepared foods by household members and overall attitude toward practicing healthy eating as measured by the Home Food Environment Helathiness Score. Surprisingly, no relationship was noted for time spent playing video games and watching television. $(1,7,8)$

\section{Conclusions}


Knowing how the environment affects our habits and the beliefs that guide us in our daily lives, it is important to keep the public aware of healthy choices. The more people who promote and lead healthy lifestyles, the more people will be inspired to make gradual changes in themselves. Participation in team sports or support groups, where the desired patterns will spread, can be an additional motivation.

An important tool of our times is social media, which used in an appropriate manner have a similar potential to change the way of thinking of the audience. An example of this is the growing body positive trend, which can unintentionally contribute to social acceptance of obesity. It is important to carefully consider all the information coming from the many sources that modern man is surrounded by. We should take care to properly verify information and motivate each other to undertake regular physical activity and make positive dietary changes. $(2,5,7,9)$

\section{References:}

1. Huang H, Yan Z, Chen Y, Liu F. A social contagious model of the obesity epidemic. Scientific Reports. 2016 Nov 28;6.

2. World Health Organization. Obesity: preventing and managing the global epidemic: report of a WHO consultation. World Health Organization; 2000. 253.

3. Tappe KA, Glanz K, Sallis JF, Zhou C, Saelens BE. Children's physical activity and parents' perception of the neighborhood environment: Neighborhood impact on kids study. International Journal of Behavioral Nutrition and Physical Activity. 2013 Mar 27;10.

4. Yang YC, Boen C, Gerken K, Li T, Schorpp K, Harris KM. Social relationships and physiological determinants of longevity across the human life span. Proceedings of the National Academy of Sciences [Internet]. 2016 Jan 19 [cited 2021 Sep 25];113(3):578-83. Available from: https://www.pnas.org/content/113/3/578

5. Christakis NA, Fowler JH. The Spread of Obesity in a Large Social Network over 32 Years A BS T R AC T [Internet]. Vol. 357, N Engl J Med. 2007. Available from: www.nejm.org

6. Vorwerg Y, Petroff D, Kiess W, Blüher S. Physical Activity in 3-6 Year Old Children Measured by SenseWear Pro ${ }^{\circledR}$ : Direct Accelerometry in the Course of the Week and Relation to Weight Status, Media Consumption, and Socioeconomic Factors. PLoS ONE. 2013 Apr 3;8(4).

7. Skinner AC, Skelton JA. Prevalence and trends in obesity and severe obesity among children in the united states, 1999-2012. JAMA Pediatrics. 2014;168(6):561-6.

8. Borrell LN, Graham L, Joseph SP. Associations of neighborhood safety and neighborhood support with overweight and obesity in us children and adolescents. Ethnicity and Disease. 2016 Sep 1;26(4):469-76.

9. Aziza R, Borgi A, Zgaya H, Guinhouya B. Simulating Complex systems: Complex system theories, their behavioural characteristics and their simulation. In: ICAART 2016 Proceedings of the 8th International Conference on Agents and Artificial Intelligence. SciTePress; 2016. p. 298-305. 